\title{
Long-time vs. short-time insemination of sibling eggs
}

\author{
JING LIU $^{1 *}$, XIAO ZHANG ${ }^{2 *}$, YUE YANG $^{1}$, JUNLIANG ZHAO $^{1}$, DAYONG HAO ${ }^{1}$, \\ JIANRUI ZHANG ${ }^{1}$, YANLI LIU ${ }^{1}$, WENBIN WU ${ }^{1}$ and XINGLING WANG ${ }^{1}$ \\ ${ }^{1}$ Reproductive Medicine Center, The Third Affiliated Hospital of Zhengzhou University, Zhengzhou, \\ Henan 450052, P.R. China; ${ }^{2}$ Cork Fertility Centre, T12 YE24 Cork, Republic of Ireland
}

Received August 18, 2015; Accepted June 15, 2016

DOI: 10.3892/etm.2016.3827

\begin{abstract}
This study aimed to evaluate the impact of a short insemination procedure on embryo development, assess the reliability of a fertilization check prior to the appearance of pronuclei and elucidate the role of rescue intracytoplasmic sperm injection (R-ICSI). Patients who had $\geq 9$ eggs, were aged $<38$ years and had normal semen samples were included. The sibling eggs of each patient were divided into two groups; one half for conventional in vitro fertilization (IVF; insemination time, $20 \mathrm{~h}$ ) and the other half for short insemination (insemination time, $5 \mathrm{~h}$ ). R-ICSI was performed where total fertilization failure (TFF) was deemed to have occurred in the short insemination group. In total, 2,465 eggs were included. No significant differences were found in fertilization, abnormal fertilization, embryo quality, clinical pregnancy and implantation rates between the short insemination and conventional insemination groups. In the short insemination group, R-ICSI was performed in 11 cycles (6.7\%); in 6 of these cycles, fertilization occurred in the patient's eggs in the $20 \mathrm{~h}$ insemination group, while in the other 5 cycles, the patient's eggs were not fertilized after $20 \mathrm{~h}$ insemination either. Following R-ICSI, 19 of 36 eggs were normally fertilized. Subsequently, 7 embryos were transferred in 4 fresh cycles resulting in one ectopic pregnancy. In conclusion, the short insemination procedure does not improve embryo development and pregnancy rates. Short insemination combined with early R-ICSI may be a method of preventing total fertilization failure, albeit with poor accuracy.
\end{abstract}

\section{Introduction}

In conventional in vitro fertilization (IVF), the incidence of total fertilization failure (TFF) has been reported to range

Correspondence to: Dr Xingling Wang, Reproductive Medicine Center, The Third Affiliated Hospital of Zhengzhou University, 7 Kangfu Street, Zhengzhou, Henan 450052, P.R. China

E-mail: xinglingwangen@163.com

*Contributed equally

Key words: short insemination, polyspermy rate, rescue intracytoplasmic sperm injection, pregnancy from 5 to $10 \%$ (1). Considering the stress and financial costs associated with fertility treatment, TFF is a catastrophic event for patients who already have been under fertility-induced pressure. Various methods have been explored to avoid this undesirable IVF result.

Rescue intracytoplasmic sperm injection (R-ICSI), in which ICSI is performed on oocytes that have failed to fertilize, has been proposed to counteract TFF. Although sporadic cases of success have been reported $(2,3)$, the effectiveness of R-ICSI is not satisfactory due to oocyte aging in vitro, that is, a delayed ICSI procedure and asynchronized endometrium $(4,5)$. In order that R-ISCI is conducted within the oocyte fertilization window, Chen and Kattera (6) removed granulosa cells from around oocytes after co-culturing the cumulus-oocyte complex (COC) with sperm for $6 \mathrm{~h}$ to determine the fertilization status. The unfertilized oocytes, defined by those being without the presence of the second polar body, were subjected to R-ICSI at $6 \mathrm{~h}$ post-insemination. This insemination method has been appraised and adopted by numerous clinics recently, not only due to the advantage of averting TFF, but also the theoretical benefit of reducing potential damage caused by sperm metabolic waste products such as free oxygen radicals $(7,8)$.

However, the efficacy of the short co-culture time with sperm remains unclear. The removal of early cumulus cells may impact embryo development (9), and the effectiveness of early R-ICSI remains debatable (10). The aim of the present study was to evaluate the impact of a short insemination time on embryo development, assess the reliability of the fertilization checking procedure prior to the appearance of pronuclei and elucidate the role of R-ICSI.

\section{Subjects and methods}

Patients. This study was a prospective cohort pair control study. From December 2013 to May 2014, a total of 165 cycles were included in the study. This study was conducted in accordance with the Declaration of Helsinki, and with approval from the Ethics Committee of the Third Affiliated Hospital of Zhengzhou University (Zhengzhou, China). Written informed consent was obtained from all participants.

The inclusion criteria were as follows: Patients had $>9$ eggs, were aged $<38$ years, and had normal semen samples according to WHO criteria 5 th edition (11). The eggs of each patient were divided into one half for conventional IVF (insemination time, $20 \mathrm{~h}$ ) and the other half for short insemination (insemination 
time, 5 h). R-ICSI was performed where TFF was deemed to have occurred in the short insemination group following the early fertilization check. Embryo transfers were divided randomly into two groups depending on whether the transferred embryos originated from the conventional or the short insemination.

Ovarian stimulation and oocyte retrieval. All patients underwent controlled ovarian stimulation using gonadotropin-releasing hormone analog (Diphreline; Ipsen, Paris, France) and the gonadotropins human menopausal gonadotropins (Lizhu Group Co., Ltd., Zhuhai, China) and Gonal-F (Merck Serono, Geneva, Switzerland). The standard or short stimulation protocols were used according to the patient's age or ovarian reserve function. Patients with antral follicle count $($ AFC) $>6$ or aged $<35$ years old were treated with the long protocol, and patients with $\mathrm{AFC} \leq 6$ or aged $\geq 35$ years old were treated with the short protocol. Drug dosage was adjusted according to ultrasound monitoring results and serum estradiol levels. Human chorionic gonadotropin (HCG; Lizhu Group Co., Ltd.) or recombinant HCG (Merck Serono, Geneva, Switzerland) was administered when $\geq 2$ follicles measured $18 \mathrm{~mm}$ in diameter. Oocyte retrieval was performed by guided transvaginal ultrasonography $38 \mathrm{~h}$ later.

Sperm preparation. Semen samples were collected by masturbation after 3-5 days of abstinence. After liquefaction, semen samples were prepared by gradient centrifugation. Finally, the sperm pellet was suspended and used for oocyte insemination.

Insemination and fertilization assessment. After egg retrieval, COCs were cut to similar sizes with insulin needles and placed in $100 \mu \mathrm{l} \mathrm{G-IVF}{ }^{\mathrm{TM}}$ (Ovoil; Vitrolife AB, Göteborg, Sweden) droplets covered by mineral oil (Ovoil; Vitrolife AB). Each oocyte was inseminated with 40,000 motile spermatozoa 2-4 h later. Cohort eggs were divided into two, with one half for conventional insemination and the other half for short insemination. Cumulus cells were removed at 5 or $20 \mathrm{~h}$ post insemination for the short insemination and conventional insemination groups, respectively. Oocytes were then placed in G-1 (Ovoil; Vitrolife AB) medium at $37^{\circ} \mathrm{C}$ in a humidified atmosphere of $6 \% \mathrm{CO}_{2}$.

An early fertilization check was conducted in the short insemination group. Oocytes were checked for the release of the second polar body and perivitelline space (PVS) following the removal of cumulus cells. Early fertilization was determined to have occurred when two distinct PBs and evident PVS were observed in an oocyte. If none of the oocytes showed the signs of early fertilization, R-ICSI was performed subsequently as previously described (6). All eggs were checked for the appearance of pronuclei at 17-20 $\mathrm{h}$ after insemination. The time sequence of the study is shown in Fig. 1.

Embryonic development and embryo transfer. Each embryo was observed daily and assessed using the following scoring system: Grade 1 , number of cells $\geq 4$ on day 2 , or number of cells $\geq 6$ on day 3 with even size blastomeres, integrated zona pellucida, regular morphology, clear cytoplasm, the absence of particles and $<5 \%$ fragments; grade 2 , number of cells $\geq 4$ on day 2 , or number of cells $\geq 6$ on day 3 with slightly unevenly size blastomeres, and 6-20\% fragments; grade 3, significantly unevenly size blastomeres, and $21-50 \%$ fragments; grade 4 , abnormal rate of embryo development, severely unequal cell size, significant cytoplasmic particles, and a great quantity of vacuole fragments (>50\%). Grades 1 and 2 were defined as high-quality embryos, and grades 1-3 were defined as utilizable embryos. Embryos were selected randomly from either the conventional or short insemination group. One or two utilizable embryos were selected for transfer. The luteal phase was supported by estradiol valerate and intramuscular progesterone injection.

Pregnancy and follow-up. Clinical pregnancy was defined as the detection of the gestational sac by ultrasound at 28 days after embryo transfer. Luteal support was continued until 10 weeks of gestation.

Statistical analysis. Statistical analysis was performed using SPSS version 17.0 statistical analysis software (SPSS, Inc., Chicago, IL, USA). Results are expressed as mean \pm standard deviation or percentage. The Chi-square test was used for comparisons of percentages and Student's t-test was used for the comparison of mean values. $\mathrm{P}<0.05$ was considered to indicate a statistically significant difference.

\section{Results}

General data. In total, there were 165 patients in this study, of which 95 had tubal factors, 19 had male factors, 4 had endometriosis, 4 had polycystic ovary syndrome, 21 had unexplained infertility and 22 had complex factors. There were $85(51.5 \%)$ patients with primary infertility and $80(48.5 \%)$ patients with secondary infertility.

From 165 egg collections, 2,465 eggs were retrieved. Cohort eggs from each egg collection were divided for the short insemination and conventional insemination groups in a principle of $50 / 50 \%$. The details of fertilization and further development parameters are summarized in Table I. No significant differences were observed in fertilization, abnormal fertilization and embryo quality.

Outcome of the two different types of insemination. When the 11 patients who underwent R-ICSI cycles were excluded, there were no significant differences in all treatment parameters between the two different types of insemination (Table II).

The embryo transfers of 69 of the 165 cycles $(41.8 \%)$ were cancelled due to elevated progesterone level, ovarian hyper stimulation syndrome or insufficient endometrial thickness. There were 201 embryos transferred in 96 cycles, of which 49 cycles involved the transfer of embryos from the short insemination group and 47 cycles were with embryos from the conventional insemination group. No significant differences were identified between the two groups in terms of age, number of eggs, number of embryos and good embryo rate. Clinical pregnancy and implantation rates were not significantly different between the patients with embryos transferred from the short insemination and conventional insemination groups (57.1 vs. $55.3 \%$, and 34.7 vs. $36 \%$, respectively; Table III). 


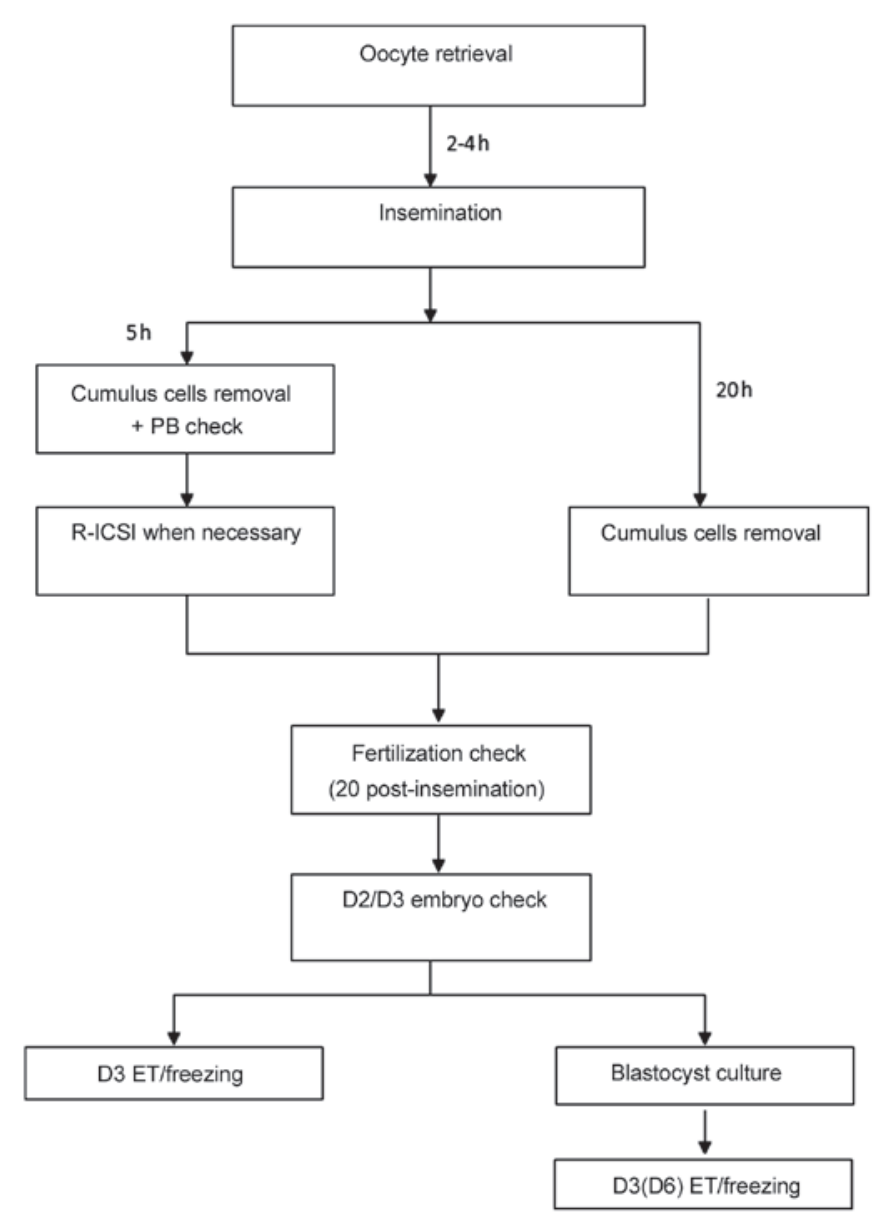

Figure 1. Time sequence for all procedures. PB, polar body; R-ICSI, rescue intracytoplasmic sperm injection; ET, embryo transfer; D, day.

$R$-ICSI cycles. In the short insemination group, R-ICSI was performed due to no oocyte activation being observed in 11 cycles $(6.7 \%)$, of which 6 cycles had fertilization of the patient's eggs in the $20 \mathrm{~h}$ insemination group. In the other 5 cycles, the patient's eggs were not fertilized after $20 \mathrm{~h}$ insemination either. Following R-ICSI, 19 of 36 eggs were normally fertilized. Subsequently, 7 embryos were transferred in 4 fresh cycles.

\section{Discussion}

Conventionally, oocytes have been co-cultured with sperm overnight, for 16-20 h. This long insemination time has been adopted as a routine practice most probably for the reason of accommodating a fertilization check in the morning of day 1 rather than to accommodate the demands of the biological clock. It has been reported that a co-incubation time as short as $30 \mathrm{sec}$ is long enough to obtain fertilization in human IVF (12). The long period of co-incubation may generate high levels of reactive oxygen species, which may damage embryos by hardening the zona pellucida and affect embryo development (13). Some reports suggest that a sperm-oocyte exposure time of 1-6 h improves the rates of fertilization, polyspermy, good-quality embryos, implantation, clinical pregnancy and ongoing pregnancy $(7,8)$. However, the advantages of a short insemination time have not been observed consistently and conflicting results have been reported (14-16). Although a meta-analysis of short insemination in IVF indicated that a reduced gamete exposure time is beneficial to clinical outcome, it also pointed out that the conclusion was compromised by design differences between studies (17).

In order to have a good control of the heterogeneity of patient characteristics, which may impact on the result of IVF treatments, sibling oocytes of each patient were assigned to either the short or the conventional insemination group in the present study. Sibling oocytes were randomized to the short insemination group or the conventional insemination group. This meant that the two groups were identical regarding factors such as etiology and the duration of the infertility, age of the women and ovarian stimulation protocol. No advantage of a short co-incubation time was found regarding fertilization rate, embryo cleavage rate or quality of the embryos, which is consistent with previous studies $(14-16,18)$. Conversely, there are some discrepancies between this study and others, showing conflicting results $(13,19)$. This could be explained by differences in the sperm density used for insemination, patient profiles, insemination method and the times of removal of cumulus cells and fertilization assessment. In comparison with the previous studies, the present study benefitted from a standard insemination protocol. A standard 100- $\mu 1$ drop size of culture media was used for insemination and 40,000 sperm for insemination, and all COCs after egg collection were trimmed to a similar size with insulin needles to control the variation introduced by the amount of sperm trapped in the cumulus. Furthermore, blastocyst culture, a less objective parameter than cleavage evaluation, was used to assess embryo quality. This study showed that the method of insemination did not affect the potential for blastocyst formation. Finally, the embryo development data were consistent with the clinical pregnancy outcomes, showing no difference between the two insemination groups, indicating that reducing the time of insemination does not improve embryo development, the embryo utilization rate or the clinical outcomes of IVF.

It has been always challenging for practitioners of artificial reproductive technology (ART) to predict fertilization results for IVF. The average fertilization rate after standard IVF is $60-70 \%$. TFF occurs in $5-10 \%$ of IVF cycles (1) and only $1-3 \%$ of ICSI cycles $(20,21)$. In part due to the advantage of the low TFF rate of ICSI, the use of ICSI increased from $39.6 \%$ of ART cycles in 1997 to $58.9 \%$ in 2004 (USA 57.5\%, Australia/New Zealand 58.6\%, Europe 59.3\%) (22). In some countries, ICSI has been considered as the only insemination method regardless semen quality (23). While considering the extra cost and the potential risk of birth defects associated with ICSI, IVF should be considered as the primary insemination method for non-male factor infertility (24). Alternatively, when conventional IVF is not successful, re-insemination of unfertilized IVF oocytes by ICSI can be considered. Since oocyte aging is so important for the outcome of re-insemination, it has been advised that R-ICSI should be performed as early as possible $(10,19,25,26)$. Chen and Kattera shortened the period of co-incubation of gametes (6). Cumulus cells were removed $6 \mathrm{~h}$ after insemination to observe the polar body status, and oocytes with no signs of activation were submitted to R-ICSI once fertilization failure was identified. 
Table I. Comparison of parameters between the two different types of insemination, including R-ICSI cycles.

\begin{tabular}{lccc}
\hline Parameter & $\begin{array}{c}\text { Short insemination } \\
\text { group }(\mathrm{n}=165)\end{array}$ & $\begin{array}{c}\text { Conventional insemination } \\
\text { group }(\mathrm{n}=165)\end{array}$ & P-value \\
\hline 2PN rate $(\%)$ & $769 / 1,227(62.7)$ & $750 / 1,238(60.6)$ & 0.286 \\
Polyspermy rate $(\%)$ & $104 / 1,227(8.5)$ & $87 / 1,238(7.0)$ & 0.179 \\
Cleavage rate $(\%)$ & $764 / 769(99.3)$ & $745 / 750(99.3)$ & 0.968 \\
Viable embryo rate $(\%)$ & $623 / 769(81.0)$ & $603 / 750(80.4)$ & 0.762 \\
High quality embryo rate $(\%)$ & $364 / 769(47.3)$ & $321 / 750(42.8)$ & 0.076 \\
Blastocyst formation rate $(\%)$ & $177 / 363(48.8)$ & $195 / 358(54.5)$ & 0.125 \\
\hline
\end{tabular}

R-ICSI, rescue intracytoplasmic sperm injection; 2PN, two pronuclei.

Table II. Comparison of parameters between the two different types of insemination, excluding R-ICSI cycles.

\begin{tabular}{|c|c|c|c|}
\hline Parameter & $\begin{array}{l}\text { Short insemination } \\
\text { group }(n=154)\end{array}$ & $\begin{array}{l}\text { Conventional insemination } \\
\text { group }(n=154)\end{array}$ & P-value \\
\hline $2 \mathrm{PN}$ rate $(\%)$ & $722 / 1,145(63.1)$ & $724 / 1,158(62.5)$ & 0.790 \\
\hline Polyspermy rate $(\%)$ & $103 / 1,145(9.0)$ & 86/1,158 (7.4) & 0.170 \\
\hline Cleavage rate $(\%)$ & $719 / 722$ (99.6) & $719 / 724(99.3)$ & 0.481 \\
\hline Viable embryo rate (\%) & $585 / 722(81.0)$ & $583 / 724(80.5)$ & 0.809 \\
\hline High quality embryo rate (\%) & $343 / 722(47.5)$ & $310 / 724(42.8)$ & 0.073 \\
\hline Blastocyst formation rate (\%) & $174 / 348(50.0)$ & $187 / 344(54.4)$ & 0.251 \\
\hline
\end{tabular}

R-ICSI, rescue intracytoplasmic sperm injection; 2PN, two pronuclei.

Table III. Comparison of the embryo transfer results from the two different insemination groups.

\begin{tabular}{lccc}
\hline Parameter & $\begin{array}{c}\text { Short insemination } \\
\text { group }(\mathrm{n}=49)\end{array}$ & $\begin{array}{c}\text { Conventional insemination } \\
\text { group }(\mathrm{n}=47)\end{array}$ & $\begin{array}{c}\text { P-value } \\
\text { Age, years }\end{array}$ \\
Clinical pregnancy rate $(\%)$ & $29.6 \pm 3.9$ & $26.5 \pm 3.9$ & 0.865 \\
Implantation rate $(\%)$ & $28 / 49(57.1)$ & $36 / 100(36.0)$ & 0.875 \\
\hline
\end{tabular}

Ages are presented as mean \pm standard deviation.

In the present study, the eggs from each patient were divided into one half for conventional insemination and the other half for short insemination combined with R-ICSI if required. There were 11 cycles in which R-ICSI was performed due to TFF occurring in the eggs with the short insemination time. In 5 out the 11 R-ICSI cycles, TFF occurred concurrently in the patient's eggs in the conventional insemination group. Four patients had R-ICSI embryos transferred. Only one of them achieved an ectopic pregnancy. It is worthwhile to mention that of the 11 R-ICSI cycles, there were 6 cycles in which ICSI was performed unnecessarily as satisfactory fertilization results were obtained in the cohort eggs from the conventional insemination. Thus, for the purpose of TFF detection, this early fertilization check has $100 \%$ sensitivity and $45.5 \%$ specificity. This indicates that the occurrence of TFF was overestimated by the early fertilization check. Although the short insemina- tion combined with R-ICSI had very high sensitivity for the prevention of TFF, the poor specificity should be noted, as such rescue attempts had the potential for tripronucleated zygote formation. Secondly, unnecessary micro-manipulation and checking might put extra stress on the eggs.

Attention to the short insemination method has been increasing, particularly in China, a developing country, where the stimulatory drugs account for up to $80 \%$ of the total IVF treatment cost, indicating that cycle cancellation for the reason of TFF may stop patients coming back for further IVF treatment due to the lack of affordability (27). The short insemination combined with R-ICSI appears to be a practical method to ease the stress from TFF. Nevertheless, this method of alleviating TFF has the disadvantages of potentially injecting fertilized eggs, unnecessary ICSI being conducted, higher polyspermy rates (28), and the requirement for extra embryologists to 
cover evening or later shifts. Considering those hidden costs, cost-effectiveness studies are required to be conducted before concluding the role of R-ICSI.

In conclusion, the present study demonstrates that a short insemination time does not improve embryo development and pregnancy rates. Short insemination combined with early R-ICSI may be a method to prevent TFF, albeit with poor accuracy.

\section{References}

1. Mahutte NG and Arici A: Failed fertilization: Is it predictable? Curr Opin Obstet Gynecol 15: 211-218, 2003.

2. Yuzpe AA, Liu Z and Fluker MR: Rescue intracytoplasmic sperm injection (ICSI)-salvaging in vitro fertilization (IVF) cycles after total or near-total fertilization failure. Fertil Steril 73: 1115-1119, 2000.

3. Lundin K, Sjögren A and Hamberger L: Reinsemination of one-day-old oocytes by use of intracytoplasmic sperm injection. Fertil Steril 66: 118-121, 1996

4. Sermondade N, Hugues JN, Cedrin-Durnerin I, Poncelet C, Benzacken B, Lévy R and Sifer C: Should all embryos from day 1 rescue intracytoplasmic sperm injection be transferred during frozen-thawed cycles? Fertil Steril 94: 1157-1158, 2010.

5. Ming L, Liu P, Qiao J, Lian Y, Zheng X, Ren X, Huang J and Wu Y: Synchronization between embryo development and endometrium is a contributing factor for rescue ICSI outcome. Reprod Biomed Online 24: 527-531, 2012.

6. Chen C and Kattera S: Rescue ICSI of oocytes that failed to extrude the second polar body $6 \mathrm{~h}$ post-insemination in conventional IVF. Hum Reprod 18: 2118-2121, 2003

7. Kattera S and Chen C: Short coincubation of gametes in in vitro fertilization improves implantation and pregnancy rates: A prospective, randomized, controlled study. Fertil Steril 80: 1017-1021, 2003.

8. Dirnfeld M, Shiloh H, Bider D, Harari E, Koifman M, Lahav-Baratz S and Abramovici H: A prospective randomized controlled study of the effect of short coincubation of gametes during insemination on zona pellucida thickness. Gynecol Endocrinol 17: 397-403, 2003

9. Wei D, Zhang C, Yin B, Wang P, Xie J, Song X, Liu Q, Hu L, Zhang Y and Hao H: Early cumulus cell removal could reduce the available embryo rate in human IVF. J Assist Reprod Genet 28: 1213-1216, 2011

10. Nagy ZP, Rienzi LF, Ubaldi FM, Greco E, Massey JB and Kort HI: Effect of reduced oocyte aging on the outcome of rescue intracytoplasmic sperm injection. Fertil Steril 85: 901-906, 2006.

11. World Health Organization: WHO Laboratory Manual for the Examination and Processing of Human Semen. 5th edition. Geneva, pp10-44, 2010

12. Bungum M, Bungum L and Humaidan P: A prospective study, using sibling oocytes, examining the effect of 30 seconds versus 90 minutes gamete co-incubation in IVF. Hum Reprod 21: $518-523,2006$

13. Gianaroli L, Cristina Magli M, Ferraretti AP, Fiorentino A, Tosti E, Panzella S and Dale B: Reducing the time of sperm-oocyte interaction in human in-vitro fertilization improves the implantation rate. Hum Reprod 11: 166-171, 1996.
14. Lundqvist M, Johansson U, Lundkvist O, Milton K, Westin C and Simberg N: Reducing the time of co-incubation of gametes in human in-vitro fertilization has no beneficial effects. Reprod Biomed Online 3: 21-24, 2001.

15. Lin SP, Lee RK, Su JT, Lin MH and Hwu YM: The effects of brief gamete co-incubation in human in vitro fertilization. J Assist Reprod Genet 17: 344-348, 2000.

16. Barraud-Lange V, Sifer C, Pocaté K, Ziyyat A, Martin-Pont B, Porcher R, Hugues JN and Wolf JP: Short gamete co-incubation during in vitro fertilization decreases the fertilization rate and does not improve embryo quality: A prospective auto controlled study. J Assist Reprod Genet 25: 305-310, 2008.

17. Zhang XD, Liu JX, Liu WW, Gao Y, Han W, Xiong S, Wu LH and Huang GN: Time of insemination culture and outcomes of in vitro fertilization: A systematic review and meta-analysis. Hum Reprod Update 19: 685-695, 2013.

18. Coskun S, Roca GL, Elnour AM, al Mayman H, Hollanders JM and Jaroudi KA: Effects of reducing insemination time in human in vitro fertilization and embryo development by using sibling oocytes. J Assist Reprod Genet 15: 605-608, 1998.

19. Zhang NY, Sun HX, Hu YL, Wang B and Xu ZP: Combination of short-period sperm-oocyte coincubation and early rescue intracytoplasmic sperm injection after total failure of in vitro fertilization. Zhonghua Nan Ke Xue 15: 538-541, 2009 (In Chinese)

20. Cheng D, Li J, Guo CC and Xiong CL: Failed fertilization after ICSI: Causes and countermeasures. Zhonghua Nan Ke Xue 17: 1131-1134, 2011 (In Chinese).

21. Flaherty SP, Payne D and Matthews CD: Fertilization failures and abnormal fertilization after intracytoplasmic sperm injection. Hum Reprod 13: 155-164, 1998.

22. Nyboe Andersen A, Carlsen E and Loft A: Trends in the use of intracytoplasmatic sperm injection marked variability between countries. Hum Reprod Update 14: 593-604, 2008.

23. Kupka MS, Ferraretti AP, de Mouzon J, Erb K, D'Hooghe T, Castilla JA, Calhaz-Jorge C, De Geyter C and Goossens V; European IVF-monitoring consortium, for the European society of human reproduction and embryology: Assisted reproductive technology in Europe, 2010: Results generated from European registers by ESHRE†. Hum Reprod 29: 2099-2113, 2014.

24. Davies MJ, Moore VM, Willson KJ, Van Essen P, Priest K, Scott H, Haan EA and Chan A: Reproductive technologies and the risk of birth defects. N Engl J Med 366: 1803-1813, 2012.

25. Liu W, Liu J, Zhang X, Han W, Xiong S and Huang G: Short co-incubation of gametes combined with early rescue ICSI: An optimal strategy for complete fertilization failure after IVF. Hum Fertil (Camb) 17: 50-55, 2014.

26. Kuczyński W, Dhont M, Grygoruk C, Pietrewicz P, Redzko S and Szamatowicz M: Rescue ICSI of unfertilized oocytes after IVF. Hum Reprod 17: 2423-2427, 2002.

27. Chambers GM, Hoang VP, Sullivan EA, Chapman MG, Ishihara O, Zegers-Hochschild F, Nygren KG and Adamson GD: The impact of consumer affordability on access to assisted reproductive technologies and embryo transfer practices: An international analysis. Fertil Steril 101: 191-198.e4, 2014.

28. Xiong S, Han W, Liu JX, Zhang XD, Liu WW, Liu H and Huang GN: Effects of cumulus cells removal after $6 \mathrm{~h}$ co-incubation of gametes on the outcomes of human IVF. J Assist Reprod Genet 28: 1205-1211, 2011. 